\title{
Rate Adaptation Mechanism for Multimedia Multicasting in Wireless Networks
}

\author{
Kandaraj Piamrat*, Adlen Ksentini ${ }^{\dagger}$, Jean-Marie Bonnin ${ }^{\ddagger}$, and César Viho ${ }^{\dagger}$ \\ *INRIA Rennes-Bretagne Atlantique, Campus Beaulieu 35042 Rennes, France \\ Telephone: (33) 299847137, Fax: (33) 299847171, Email: kandaraj.piamrat@inria.fr \\ ${ }^{\dagger}$ IRISA/University of Rennes I, Campus Beaulieu 35042 Rennes, France \\ Telephone: (33) 299847416, (33) 299847142, Fax: (33) 299847171, Email: \{adlen.ksentini, cesar.viho\}@ irisa.fr \\ $\ddagger$ Institut Telecom/Telecom Bretagne, CS-17607, 35576 Cesson-Sévigné, France \\ Telephone: (33) 299127007, Fax: (33) 299127030, Email: jm.bonnin@telecom-bretagne.eu
}

\begin{abstract}
Nowadays, wireless networks have been deploying everywhere, with IEEE 802.11 as the most popular standard. However, wireless resources are scarce and wireless condition varies often. These limitations are crucial for applications with tight $Q \circ S$ requirements such as video or voice over IP. To cope with the problems, the standard has provided many features including multi-rate capability, which is the focus of this paper. Multi-rate capability is beneficial especially for multicast transmission, in which the traffic sent by default at basic rate may result in capacity wasting because of longer channel occupancy. Moreover the lack of feedback mechanism makes it difficult to deal with reliability or service quality. Some protocols have proposed to use rate adaptation to handle the problems but none of them takes into account quality of experience (QoE), which is an essential quality indicator in multimedia traffic, for making decision. Therefore, we propose in this paper a novel rate-adaptation mechanism based on quality of experience. We make use of PSQA (Pseudo-Subjective Quality Assessment) tool for obtaining mean opinion score in real-time. Our objective is to improve bandwidth utilization while satisfying user experience. We illustrate significant performance improvement obtained by our scheme according to this goal.
\end{abstract}

Index Terms-Rate Adaptation, Multicast, Video Streaming, Wireless Networks, Quality of Experience.

\section{INTRODUCTION}

Multicast over wireless networks is a fundamental communication function because wireless network is inherently broadcast-oriented. This means that a packet can be intercepted by all nodes in the sender's transmission range. Hence, each packet is sent just once and will reach all intended recipients in the multicast group. Therefore, multicast is an efficient method to transmit same data to a group since it allows transmission of data to multiple destinations using fewer network resources. Moreover, the fast-growth of wireless network and its application has pushed the deployment of multicast communication over wireless networks. Various applications support multicast, for example, conference meeting, mobile commerce (mobile auctions), military command and control, distance education, entertainment service, and intelligent transport systems.

However, multicast application has some constraints. Multicast traffic has been set to the lowest transmission rate (basic rate) in order to reach all mobile nodes especially the further ones because they are subject to important signal fading and interference. The lower rates disadvantage transmission in terms of channel occupancy since they take longer time than the higher rates to send same amount of information. This performance anomaly has been presented in [1], it is mentioned that slow host may considerably limit the throughput of other hosts roughly to the level of lower rate. Another constraint in multicast transmission is the lack of acknowledgment (ACK) and retransmission due to huge amount of traffic overhead these packets will generate. This is severe when transmission mode is multicast. First, the number of ACK will be multiplied by the number of recipients in the multicast group, which could cause collision due to ACK implosion. Second, it is difficult to deal with synchronization in the group if sender has to handle retransmission with per-connection basis.

Besides, multicast transmission is not reliable, losses can occur. In wireless networks, there are principally two types of losses due to two factors: Bit Error Rate (BER) resulted from signal strength and physical modulation, it can be called as channel error; and Collision resulted mainly from congestion in the network. It can be noticed that rate adaptation, a mechanism that switches transmission rate to improve performance, is not efficient in lossy network caused by the second factor as the network is already high-loaded and it would not perform better if we slower down the transmission. On the other hand, rate adaptation can be helpful in the first case when losses are caused by BER due to bad channel condition (path loss, interference, distance, etc). Therefore, this paper deals mainly with this type of loss, which is essential in wireless multicast communications.

As to improve performance of multicast transmission (loss rate, network utilization, and user perception), we apply rate adaptation mechanism using quality of experience as an indicator for transmission rate selection. Quality of Experience or QoE [2] is a new concept, which is more appropriate to deal with multimedia service such as video or voice over IP. With these types of applications, quality of service is hardly determined only by technical parameters such as BER, SNR, etc... It makes more sense to evaluate quality by users' opinion on their perception of the application that is why it is called Quality of Experience. This metric can be evaluated in terms of Mean Opinion Score (MOS) shown in Table I. 
TABLE I

MEAN OPINION SCORE - MOS

\begin{tabular}{|c|c|c|}
\hline MOS & Quality & Impairment \\
\hline \hline 5 & Excellent & Imperceptible \\
\hline 4 & Good & Perceptible but not annoying \\
\hline 3 & Fair & Slightly annoying \\
\hline 2 & Poor & Annoying \\
\hline 1 & Bad & Very annoying \\
\hline
\end{tabular}

However, it is difficult to ask people to evaluate the score and then adjust the transmission rate in real-time. The evaluation procedure is very complex and time-consuming, it also needs manpower. Thus it is not practical for real-time usage. For these reasons, in this paper we use Pseudo Subjective Quality Assessment (PSQA) [3], a real-time QoE assessment tool based on Random Neural Network, to evaluate QoE and we adapt transmission rate accordingly.

The rest of this paper is organized as follow. We begin by giving description of related works concerning rate adaptation mechanism in IEEE 802.11 wireless networks in Section II. We continue with the proposed scheme based on quality of experience in Section III. We explain simulation setup such as implementation, scenario, and threshold selection in Section IV. The results are provided in Section V where we also give comparisons of our schemes to others existing ones. Finally, we give conclusions and future works in Section VI.

\section{RELATED WORK}

In this section, we begin by giving some backgrounds concerning rate adaptation mechanisms in IEEE 802.11 [4] unicast namely Auto Rate Fallback (ARF), Receiver based Auto Rate (RBAR), and Adaptive ARF (AARF). Then we continue with rate adaptation mechanisms designed especially for multicast in wireless networks such as Rate Adaptive Multicast (RAM) and SNR-based Auto Rate for Multicast (SARM).

\section{A. Rate adaptation mechanisms in IEEE 802.11}

In IEEE 802.11, the signal strength or SNR (Signal to Noise Ratio) obtained at receiver is linked to transmission rate of access point. However, when distance between the access point and receiving stations increases, the reception condition degrades (due to interference, obstacles etc...), hence the need to switch to another lower rate. We describe three rate-adaptation protocols for unicast environment as follow.

- ARF-Auto Rate Fallback

In ARF protocol [5], when SNR decreases, an access point tries to recover by decreasing the bandwidth from $11 \mathrm{Mbps}$ to 5.5, 2, and $1 \mathrm{Mbps}$ respectively. In fact, the access point switches to a higher rate when a certain number (ten) of packets has been successfully received; it switches back to the lower rate when a failure occurs right after the increase. If a failure occurs when the number of consecutive successful transmissions is less than ten, the access point switches to a lower rate only after two consecutive failures.

\section{- RBAR- Receiver-Based Auto Rate}

RBAR [6] has the goal of performance optimization in wireless networks using also a rate adaptation mechanism at MAC layer. In RBAR protocol, RTS/CTS (Request/Clear To Send) mechanism is enabled in order to get/send feedback from receiver. In fact, RTS is sent out before each transmission by the sender and it is received by the receiver who computes the SNR of the frame. After that, the receiver compares the received SNR with the mappings between transmission rate and SNR in Table II, then it sends back the transmission rate for the sender to use in the next transmissions in CTS. RTS and CTS headers have been modified for the purposes. This mechanism is based on SNR (computed with a priori channel model), which is a physical parameter that does not always correlate well with human perception. Moreover, RTS/CTS mechanism is not enabled in multicast.

- AARF-Adaptive ARF

In AARF [7], the authors also use threshold-based mechanism but instead of setting it to a fixed number, the threshold follows binary exponential backoff continuously at runtime to better reflect to the channel conditions. This means they multiply by two the number of consecutive successful transmission required to switch to a higher rate. The effect of this adaptation mechanism is to increase the period between successive failed attempts to use a higher rate. With fewer failed transmissions and retransmissions, the overall throughput is improved. Even though AARF is an efficient adaptation mechanism, unfortunately it cannot be applied to multicast scenario since the implementation of rate adaptation also includes the existence of acknowledgment and retransmission, which are disabled in multicast communication.

\section{B. Rate adaptation in wireless multicast}

Rate adaptation mechanism in multicast is different than in IEEE 802.11 unicast. The critical concern is the lack of feedback mechanism from receivers since there is neither acknowledgment (ACK) nor negative acknowledgment (NACK). Moreover, there is no retransmission to recover from loss/error. Many researchers have proposed reliable multicast protocols such as [8] or [9] to deal with unreliability issue in multicast. Another important issue is performance of multicast due to the fact that multicast traffic is set to basic rate. Few papers deal with this problem using multi-rate capability available in IEEE 802.11. We give brief descriptions of two of previous works here.

- RAM-Rate Adaptive Multicast

In this protocol [10], the idea is similar to RBAR because multicast receivers make use of RTS to measure channel condition and send back transmission rate for sender to use in CTS. In case that a member does not receive the data frame correctly, it will send a NACK (Not Acknowledge). For enhancing the throughput, the authors added a frame sequence field to RTS. This field is used by the member to check whether multicast data frame is a new 
frame or retransmission. If a frame is a retransmission of a previously successfully received frame, a member will not participate in this multicast transmission. This reduces the number of retransmission. It can be noticed that the protocol makes use of RTS/CTS and NACK, which are disable in multicast. Moreover, there are many modifications to existing frames.

- SARM-SNR-based Auto Rate for Multicast

Park et al. have proposed SARM (SNR-based Auto Rate for Multicast) [11], a MAC-layer multicast mechanism with a multi-rate transmission. By changing multicast transmission rate on the basis of SNR values reported by mobile nodes, the wireless channel is used more efficiently than the default rate. In fact, SARM adapts a transmission rate according to the SNR of the node experiencing the worst channel condition. The SNR and rate mapping references are obtained from Table II. This table (SARM columns) lists transmission rates and the required SNRs for PSNR to be higher than 30 representing good quality at user-end. Due to the lack of feedback mechanism in multicast, the authors propose a channel probing mechanism to inform the access point of the channel quality at mobile nodes. To avoid collision when nodes transmit feedback to the access point, the author also proposed a backoff timer for each mobile node based on the received SNR. This scheme seems to have the closest goal to ours (good user-end quality), we decided to compare our performance with it in Section V.

TABLE II

SNR-TRANSMISSION RATE MAPPING TABLE

\begin{tabular}{|c||c|c|c|}
\hline $\begin{array}{c}\text { Rate } \\
\text { (Mbps) }\end{array}$ & RBAR & $\begin{array}{c}\text { SARM with } \\
\text { FCS OFF }\end{array}$ & $\begin{array}{c}\text { SARM with } \\
\text { FCS ON }\end{array}$ \\
\hline 11 & 30 & 26 & 30 \\
5.5 & 25 & 21 & 24.5 \\
2 & 21 & 17.5 & 21 \\
\hline
\end{tabular}

It can be seen that previous works always handle the problem at packet level using technical parameters as metric to decide whether to switch up or down. In this paper we propose to handle the problem at user perception level as we explain more details in the next section.

\section{The Proposed SCHEME}

We propose a novel rate adaptation mechanism that adjusts transmission rate according to user-end perception in terms of quality of experience. The idea of the proposed scheme is to use QoE feedback from mobile stations to provision the current condition of the network and then adapt the rate accordingly. For communications between access point and mobile nodes, we make use of IEEE $802.11 \mathrm{k}$ standard [12] since it has specified many measurement requests and reports that can be used. It can be noticed that with IEEE $802.11 \mathrm{k}$ measurements, our control traffic is not significant in terms of overhead as control traffic is sent much less frequently than other packet-level schemes. For example, control traffic is sent every second in our scheme comparing to every single packet in the other schemes.

\section{A. Pseudo-Subjective Quality Assessment}

In order to get QoE in real-time, we deployed PSQA (Pseudo-Subjective Quality Assessment) [3], which is based on statistic learning using random neural network (RNN). The idea is to train the RNN to learn the mapping between QoE scores and technical parameters so that we can use a trainedRNN as a function to give QoE score in real-time. In order to use this tool, three steps need to be done beforehand. We summarize them as follow.

1) Configuration: we first choose configurations, which are sets of quality affecting parameters such as codec, bandwidth, loss, and delay along with their ranges of values that will be used for the RNN training. Then we take several video sequences to be distorted with the configurations previously chosen.

2) Training: we ask for a panel of human observer to evaluate the distorted videos (Fig. 1) and then we store the configurations and corresponding MOS into two databases: training and validation databases. After that, we train the RNN to learn the mapping of configurations and scores as defined in the training database. Once the tool has been trained, we have a function $f()$ that can map any value of parameters into MOS.

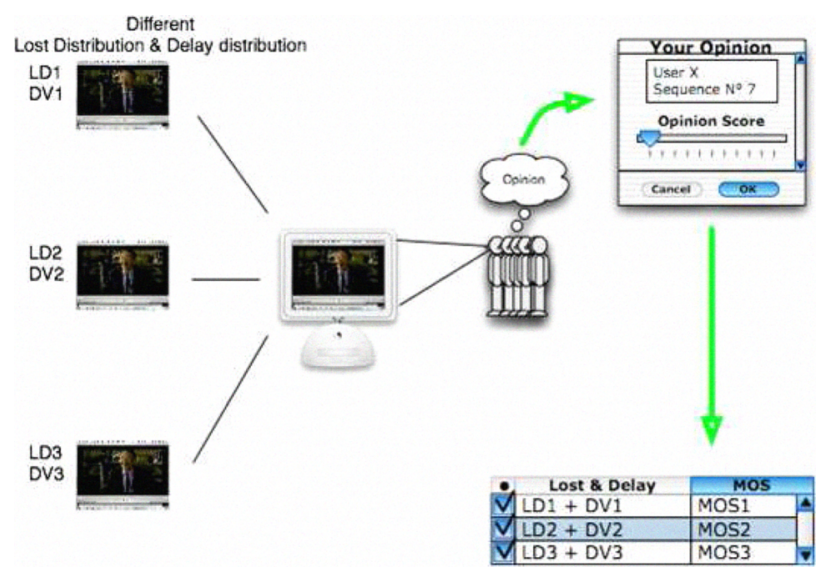

Fig. 1. Subjective Quality Assessment

3) Validation: trained RNN is validated by comparing value given by the $f()$ at the point corresponding to each configuration in the validation database. If the values are close enough, the RNN is validated; otherwise, we have to review chosen configurations.

Once RNN has been validated, PSQA can be used anywhere in real-time without interaction from human. It needs values of technical parameters as input and it gives scores (in MOS) as if there were real humans marking the playing media.

For this scheme, we deployed PSQA that have been trained and validated using statistics of application frame level (I/P/B) to map with users' perception. A configuration used for evaluation is composed of the following parameters: loss rate of the I frame, loss rate of the $\mathrm{P}$ frame, loss rate of the B frame, and mean loss burst size of the I frame. The last parameter is used for capturing the way losses are distributed in the flow as it affects dramatically the perception of video [13]. 


\section{B. Algorithm}

We propose to use QoE as indicator for switching from one transmission rate to another. This is because we found out that for multimedia application such as video multicasting, it is more reasonable to adapt the transmission rate taking into account the quality perceived at the user end. We assume PSQA running on every multicast node.

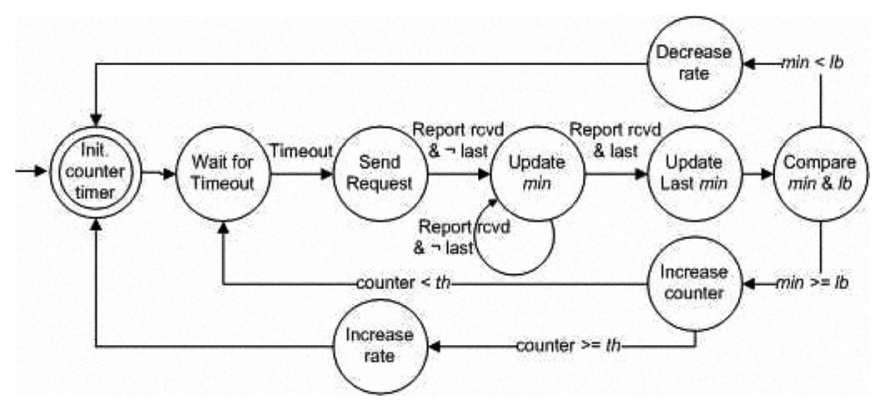

Fig. 2. Access point behavior during multicast session

We describe in Fig. 2 the behavior of an access point in our scheme during multicast session. At the beginning, the access point transmits multicast traffic at its highest rate. The AP monitors its attached clients every monitoring interval (mi). Note that our scheme uses time scale in terms of second because this scale is more reasonable than scaling in packet when dealing with human perception. When the timer rings, AP begins by sending requests to multicast members in order of membership precedence. This is to avoid collision of reports sending back from members. When a report is received, AP updates the minimum MOS ( $\mathrm{min}$ ) of the group accordingly. Once the last report has been received, it compares $\min$ with the lower bound $(l b)$. This $l b$ is computed by adding a margin $(m g)$ to a reference score $(r f)$, which is an acceptable score for the application. If $\min$ is less than $l b$, then AP switches immediately to one-step lower rate until minimum rate. If $\min$ is higher than $l b$, then AP increases the counter (representing the duration that AP has been waiting). If the counter reaches a threshold $(t h)$, then AP switches to one-step upper rate until maximum rate.

It can be noticed that when condition degrades, the access point in our scheme lowers the transmission rate immediately. This is to adapt instantly to bad condition because it is essential to recover from the bad situation rapidly. When network condition becomes better (i.e $\min$ is higher than $l b$ ) for a certain number of times, the AP switches to higher rate. This waiting threshold is used to avoid ping-pong effect; before sending at higher rate (higher risk of BER), we make sure that this condition remains quite stable.

\section{Simulation Setup}

In this section we begin by description of our scenario. We continue with explanations of the implementation of our scheme in NS2. Then we describe how we have selected the value for the threshold mentioned in our algorithm.

\section{A. Scenario}

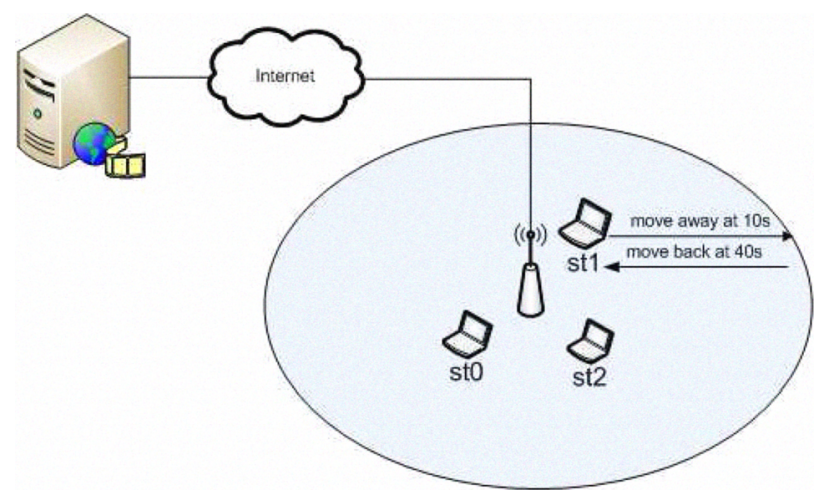

Fig. 3. Topology of our scenario

Fig. 3 illustrates our topology; there is one video server on the Internet with multicast nodes connected to it via an access point. For our test, we use video with encoding rate illustrated in Fig.4. According to the video encoding rate and limited bandwidth in basic rate, we do not put many multicast nodes in order not to be biased concerning throughput issue. We decided to test with three multicast nodes. At the beginning, all nodes locate near by the access point (less than $50 \mathrm{~m}$ radius). At $10 \mathrm{~s}$, station 1 (st1) moves away from the access point $(150 \mathrm{~m})$, and then at 40s it begins to move back to its initial position.

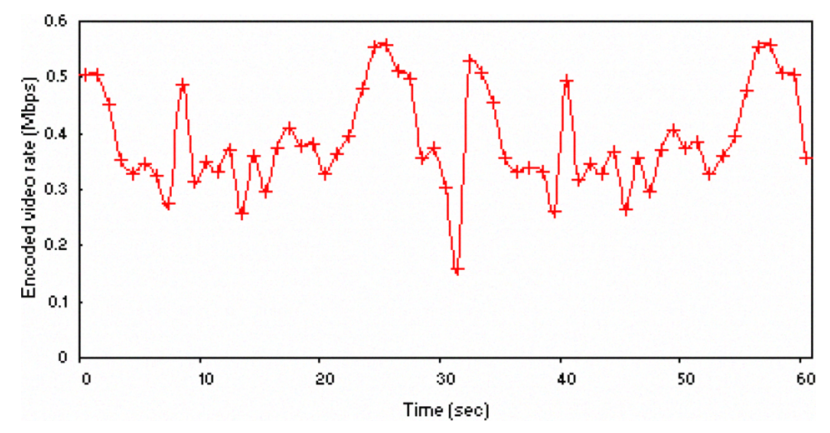

Fig. 4. Rate variation of encoded video

\section{B. Implementation}

We are interested in wireless networks IEEE 802.11 operating in infrastructure mode meaning that all traffic passes through an access point. The video sequence is an H.264coded sequence of duration 60 seconds. It is encoded at $384 \mathrm{Kbps}$ and streamed in multicast mode using UDP. Our implementation has been done via the network simulator NS2 version 2.29 [14]. We patch wireless IEEE 802.11 implementation flaws of the original version with wireless update patch from Fiore [15]. The patch includes realistic channel propagation, Ricean propagation model, 802.11 bug fixes, multiple data transmission rates support. We implement video streaming application by adding a video packet transmission module in NS-2. For communications between PSQA and NS2, we have integrated PSQA into NS2 so that it can get required statistics input for RNN. We adapt modulation in realtime according to PSQA score using our algorithm. 


\section{Threshold Selection}

We simulate different values in order to get the best threshold. Knowing that $m i$ is set to 1 second, we select to test 8 different values ranging from 1 to 8 . We illustrate in Fig. 5 and Fig. 6 the quality of experience and the goodput obtained with different values of threshold. Please note that the curves in Fig. 5 are normalized, this means that the results are divided by maximum value which is MOS $=5$. Both graphs are stacked meaning that the values have been shifted by $x$ which is equal to $i-1$ where $i$ is the value of threshold.

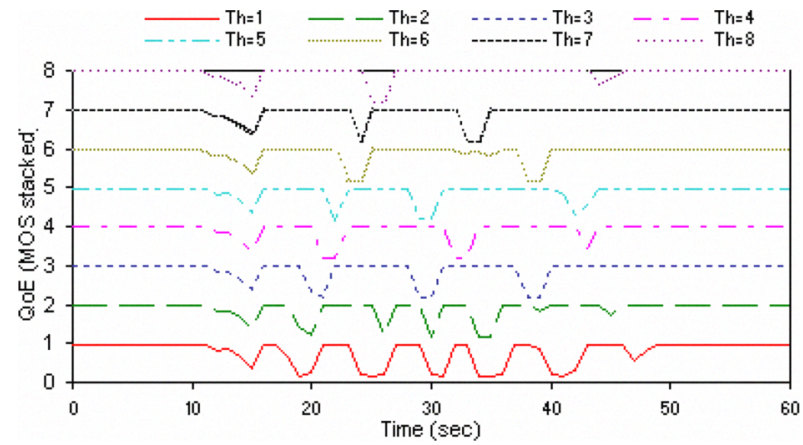

Fig. 5. Quality of experience for different values of threshold

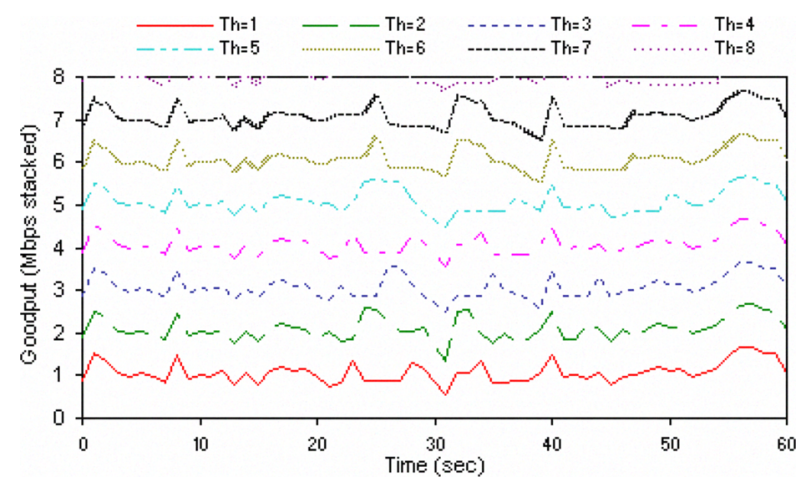

Fig. 6. Goodput for different values of threshold

Because the curves have similar trends we also give summaries of average QoE and goodput values (of all connections) for each threshold in Table III(a). We have found that surprisingly the goodput variation is not effected that much if we consider the whole connection duration. So we focused on the duration while one node is in movement (during 20s to 40s) and we can see the different as shown in Table III(b).

TABLE III

QOE AND GOODPUT OF DIFFERENT THRESHOLDS

(a) for the whole connection

\begin{tabular}{|c|c|c|c|c|c|c|c|c|}
\hline Threshold & $\mathbf{1}$ & $\mathbf{2}$ & $\mathbf{3}$ & $\mathbf{4}$ & $\mathbf{5}$ & $\mathbf{6}$ & $\mathbf{7}$ & $\mathbf{8}$ \\
\hline MOS & 3.85 & 4.38 & 4.40 & 4.48 & $\mathbf{4 . 4 8}$ & 4.51 & 4.59 & 4.61 \\
\hline Goodput & 1.05 & 1.07 & 1.04 & 1.05 & $\mathbf{1 . 0 6}$ & 1.07 & 1.07 & 1.05 \\
\hline
\end{tabular}

(b) during mobility

\begin{tabular}{|c|c|c|c|c|c|c|c|c|}
\hline Threshold & $\mathbf{1}$ & $\mathbf{2}$ & $\mathbf{3}$ & $\mathbf{4}$ & $\mathbf{5}$ & $\mathbf{6}$ & $\mathbf{7}$ & $\mathbf{8}$ \\
\hline MOS & 2.98 & 3.79 & 3.8 & 4.13 & $\mathbf{4 . 3 2}$ & 4.08 & 4.32 & 4.51 \\
\hline Goodput & 0.98 & 1.05 & 0.97 & 0.98 & $\mathbf{1 . 0 5}$ & 1.05 & 1.05 & 1.06 \\
\hline
\end{tabular}

With all the arguments observed from the experiments, we have selected value 5 for $t h$ because it is a compromised value that gives reasonable reactivity while giving high MOS and goodput. Therefore, the simulations in the next section have been conducted with configurations in Table IV.

TABLE IV

CONFIGURATION OF OUR PARAMETERS

\begin{tabular}{|c||c||c|}
\hline Parameter & Description & Value \\
\hline $\mathrm{mi}$ & monitoring interval & 1 \\
$\mathrm{th}$ & threshold & 5 \\
$\mathrm{rt}$ & reference score & 3 \\
$\mathrm{mg}$ & margin & 1 \\
$\mathrm{lb}$ & lower bound & 4 \\
\hline
\end{tabular}

\section{RESULts}

We illustrate the results with two metrics: the goodput (for network utilization) and QoE (for user perception). We compare our scheme to the default multicast (1Mpbs), maximum throughput (11Mbps), and SARM-like mechanism. We decided to compare our performance with SARM because our objectives are similar. We both want to guarantee quality of service at the receiver; SARM makes use of SNR and PSNR, our scheme makes use of QoE.

\section{A. Goodput}

We first illustrate in Fig.7 the average goodput of all stations obtained from each scheme. Then we detail to see how an individual station behaves in terms of goodput. For that we show two more graphs concerning a fixed station (st0) located near by the AP in Fig. 8 and a mobile station (moving away from and back to the AP) in Fig. 9.

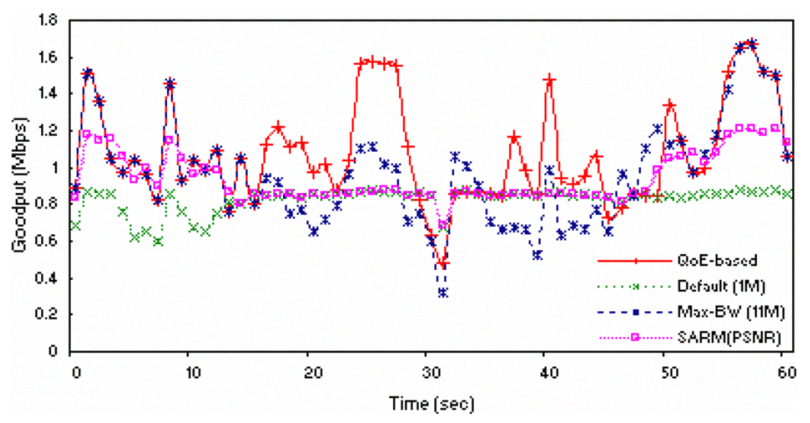

Fig. 7. Average goodput of all stations

Observation from Fig.7:

- It can be seen that our scheme provides highest average goodput. More importantly, when the node moves (during 10 s to 40 s), our average goodput is much higher than all others. This is because the scheme has adapted directly to user perception resulted from several parameters.

- When transmit at default rate (1 Mbps), throughput is the lowest in general (graph before 10s and after 40s). This proves the problem of bandwidth wasting in multicast.

- Using maximum rate gives high goodput at the beginning and at the end. However, when the distance increases 
(with mobility), channel condition degrades and this strategy performs badly.

- SNR-based performs better than default multicast rate, which is conformed to what have been mentioned in [11]. However, SNR in our scenario is quite low because of mobility and this makes the scheme change to lowest rate as we can observe in the graph; when the mobile station begins to move, the scheme behaves the same way as in default-1M.

We can see from Fig.8 that for a fixed station located nearby the AP, its goodput does not change that much among different schemes. The variation is due more to the encoding rate (shown in Fig.4) than the channel condition. However, we can observe that using $11 \mathrm{M}$ for transmission gives a little higher advantage in terms of goodput. This is because when the station is close to the AP, it can profit efficiently from short distance and high transmission rate.

On the contrary, for a moving station in Fig.9 its goodput varies often during station's movement. We observe few drops in our scheme due to the time used to switch to lower rate. We also observe that using high transmission rate (11M) giving very bad results; this is due to the high BER the station suffered when moving away from and back to the AP.

Note here that we illustrate in this paper only the goodput performance of multicast traffic. It can be noticed that if we consider also background traffic, its goodput will be increased when the rate increases and we gain more goodput as much as we stay at higher rates. This can be explained by the fact that sending at faster rate allows more time slots for other traffics. The rate variation of our scheme is presented in Fig.10.

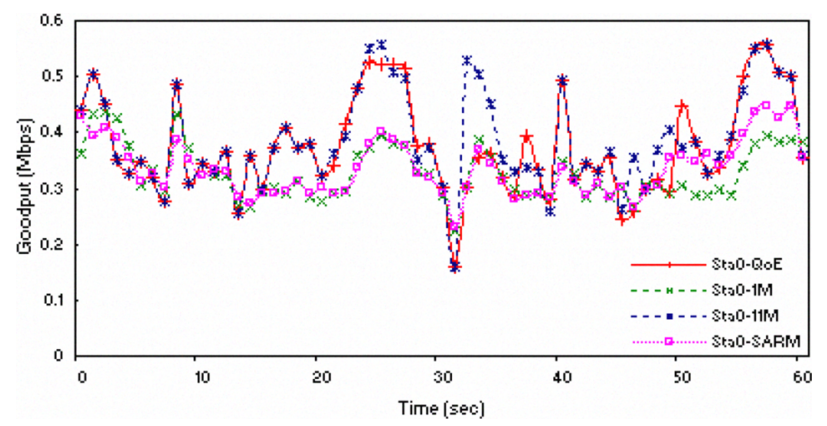

Fig. 8. Goodput of a fixed station

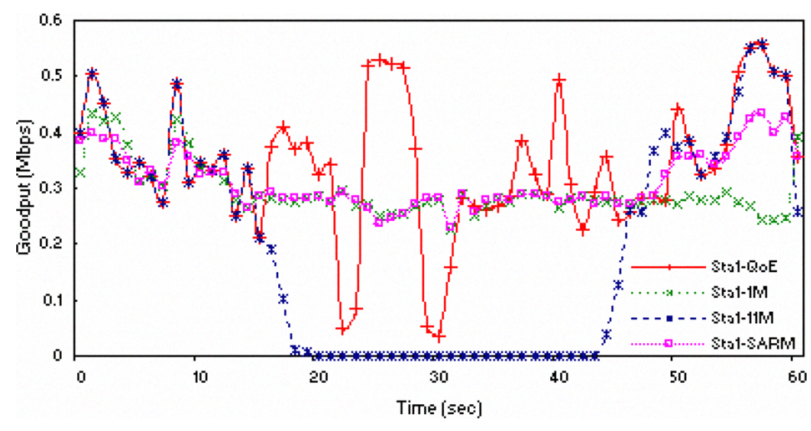

Fig. 9. Goodput of a moving station

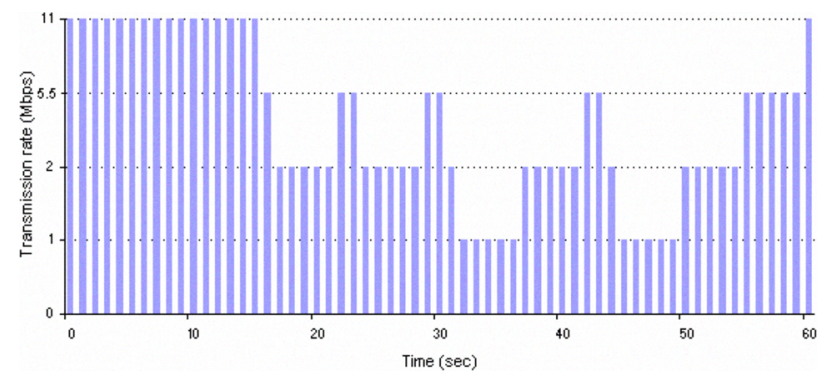

Fig. 10. Rate adaptation of our scheme during the test scenario

\section{B. Quality of Experience}

We illustrate two graphs concerning minimum QoE in time and average QoE of all stations.

Fig. 11 illustrates the scores obtained by a member encountered the worst channel condition. It can be seen that our scheme outperforms the others. During moving period, we can see that all schemes experience quite bad performance. The worst scheme is maximum-11M because the rate is too high, and then follows by SNR-based and Default-1M respectively. Despite that our scheme performs the best, we also have some drops caused by the time taken to adapt to the bad channel condition.

Fig. 12 illustrates the overall performance of the network. Since we use QoE as indicator in our scheme, we get a great performance in terms of QoE (the average QoE is at least 3.5). However, there are a few drops in the graph due to the time our scheme uses to adapt to the new condition. We also observed that the main problem of SARM-like mechanism may be caused by PSNR definition that does not have a direct relationship with QoE.

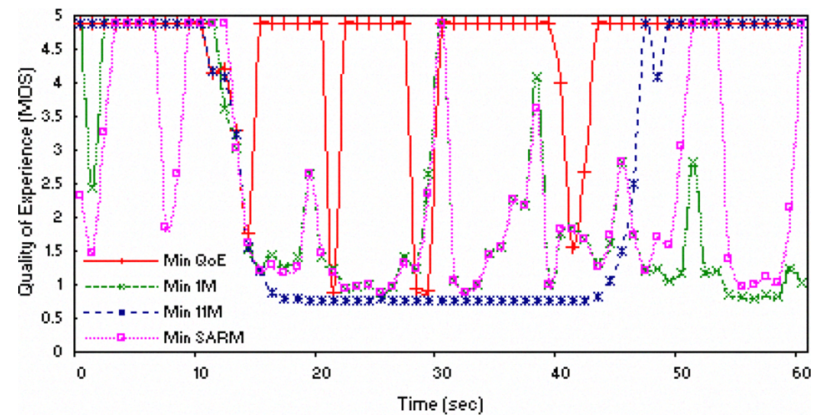

Fig. 11. Minimum QoE during multicast for each scheme

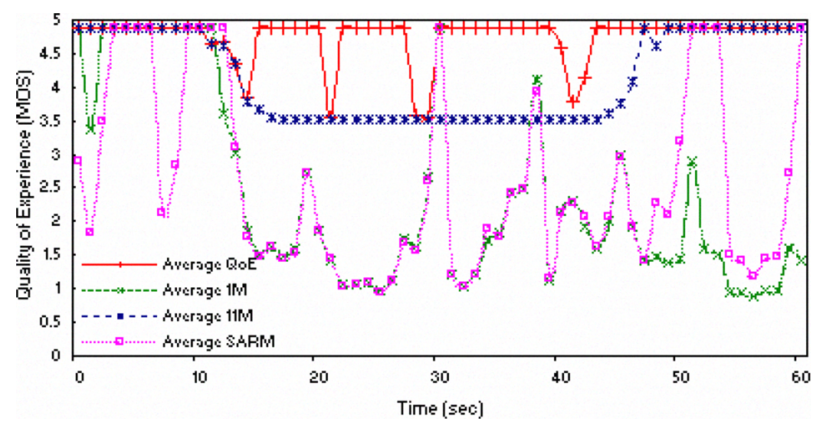

Fig. 12. Average QoE of all stations for each scheme 


\section{CONCLUSIONS AND FUTURE WORKS}

We have proposed a novel rate adaptation mechanism, which is based on quality of experience at the receiver. This is because we found out that the quality of experience is the most important metric for multimedia applications. We decided to use our mechanism to treat multicast performance problem because all losses in multicast mainly resulted from channel error. Thus, adapting rate can help improving network performance. We have tested different values for selecting the best threshold. Then we have shown that our scheme outperforms the other schemes in terms of network utilization (goodput) and user satisfaction (quality of experience).

It can be noticed that for mechanism like rate adaptation, the method to select the threshold is very important. In this paper, we have investigated the fixed threshold based approach with quality of experience as metric for changing transmission rate and we have obtained good results. However, the value of threshold needs to be chosen carefully in order to obtain a good performance. In the future, we plan to investigate deeper on the threshold selection deploying adaptive approach in order to better adjust threshold to the varying network environment.

\section{REFERENCES}

[1] M. Heusse, F. Rousseau, G. Berger-Sabbatel, and A. Duda, "Performance anomaly of $802.11 \mathrm{~b}$," INFOCOM, Twenty-Second Annual Joint Conference of the IEEE Computer and Communications Societies, vol. 2, pp. 836-843, March-3 April 2003.

[2] ITU, "Definition of Quality of Experience (QoE)," Reference: TD 109rev2 (PLEN/12), January 2007.

[3] G. Rubino, "Quantifying the Quality of Audio and Video Transmissions over the Internet: the PSQA Approach," in: Design and Operations of Communication Networks: A Review of Wired and Wireless Modelling and Management Challenges Edited by J. Barria, Imperial College Press, 2005.

[4] "IEEE 802.11, Part 11: Wireless LAN Medium Access Control (MAC) and Physical Layer (PHY) Specifications," IEEE Std 802.11-2007 (Revision of IEEE Std 802.11-1999), pp. C1-1184, 12 June 2007.

[5] A. Kamerman and L. Monteban, "WaveLAN-II: A high-performance wireless LAN for the unlicensed band," Bell Labs technial journal, vol. 2, no. 3, 1997.

[6] G. Holland, N. Vaidya, and P. Bahl, "A rate-adaptive mac protocol for multi-hop wireless networks," in MobiCom:Proceedings of the 7th annual international conference on Mobile computing and networking, New York, NY, USA.

[7] M. Lacage, M. H. Manshaei, and T. Turletti, "IEEE 802.11 rate adaptation: a practical approach," in MSWiM: Proceedings of the 7th ACM international symposium on Modeling, analysis and simulation of wireless and mobile systems, New York, NY, USA.

[8] J. Kuri and S. Kasera, "Reliable multicast in multi-access wireless LANs," INFOCOM'99. Eighteenth Annual Joint Conference of the IEEE Computer and Communications Societies. Proceedings. IEEE, vol. 2, pp. 760-767 vol.2, Mar 1999.

[9] M.-T. Sun, L. Huang, A. Arora, and T.-H. Lai, "Reliable MAC Layer Multicast in IEEE 802.11 Wireless Networks," in ICPP '02: Proceedings of the 2002 International Conference on Parallel Processing (ICPP'02). Washington, DC, USA: IEEE Computer Society, 2002, p. 527.

[10] A. Basalamah, H. Sugimoto, and T. Sato, "A Rate Adaptive Multicast Protocol for Providing MAC Layer Reliability in WLANs," IEICE Transactions on Communications, vol. E89-B, no. 10, 2006.

[11] Y. Park, Y. Seok, N. Choi, Y. Choi, and J.-M. Bonnin, "Rate-adaptive multimedia multicasting over IEEE 802.11 wireless LANs," CCNC: Consumer Communications and Networking Conference, 2006. CCNC 2006. 3rd IEEE, vol. 1, pp. 178-182, 8-10 Jan. 2006.

[12] "IEEE 802.11k, Part 11: Wireless LAN Medium Access Control (MAC) and Physical Layer (PHY) Specifications Amendment 1: Radio Resource Measurement of Wireless LANs," pp. c1-222, 12 June 2008.

[13] H. Cancela, P. Rodriguez-Bocca, and G. Rubino, "Perceptual quality in p2p multi-source video streaming policies," Global Telecommunications Conference, 2007. GLOBECOM '07. IEEE, pp. 2780-2785, Nov. 2007.

[14] "The Network Simulator - NS-2," http://www.isi.edu/nsnam/ns/.

[15] Marco Fiore, "NS-2.29 Wireless Update Patch," Electronics Department, Politecnico di Torino, Italy, http://www.tlc-networks.polito.it/fiore/. 